\section{GASTRO-INTESTINAL STUDIES}

v. THE PROTEIN CURVE OF GASTRIC DIGESTION IN NORMAL AND PATHOLOGIC CASES *

\section{J. ALEXANDER CLARKE, JR., A.B.} AND

MARTIN E. REHFUSS, M.D. PHILADELPHIA

TECHNIC OF THE REACTION AND FALLACIES OF THE TEST

Within recent years considerable interest has been shown in the study of the protein content of the gastric juice owing to the application of methods for the determination of this content in arriving at a differential diagnosis of carcinomā of the stomach and benign achylias. But little interest was manifested in the importance of the protein content in other conditions in which the acidity was present or pronounced. Furthermore, all methods which were evolved toward a solution of this problem considered the protein content merely from the point of view of a single phase in the gastric cycle. By means of a method recently devised by one of us, it has been possible to study the protein content systematically in every phase of digestion and to obtain data of importance. We have made a study of the principles underlying the reaction and shall endeavor to point out the fallacies connected with the ordinary method of examination. The regular Wolff technic ${ }^{1}$ was used with slight modifications, but instead of testing the protein content at the one-hour interval, we tested specimens $^{2}$ throughout the entire period of digestion.

Readings were immediately made and the tube giving a ring at the greatest dilution of gastric juice was recorded. ${ }^{3}$ In every instance it was desirable to perform the test under conditions which were as constant as possible, and for that reason readings were always made against a dark background. If the tubes were left standing for fifteen minutes or longer it was remarked that solutions of even greater dilution than the one recorded were capable of giving a ring. The importance of immediate examination is therefore insisted on. A second point should be brought out.

* From the Laboratory of Physiological Chemistry of the Jefferson Medical College.

1. Wolff: Magen und Darmkrankheiten, Berlin, 1912, p. 217.

2. These specimens were obtained by means of the fractional tube which one of us (Rehfuss: Am. Jour. Med. Sc., June, 1914, p. 848; New York Med. Jour., Aug. 22, 1914. Rehfuss, Bergeim and Hawk, THE JOURNal A. M. A., Sepi. 12, 1914, p. 909) devised. Specimens were obtained in this way at fifteen-minute intervals, and at five-minute intervals during the first fifteen minutes. One c.c. cf the filtered juice was then diluted with 9 c.c. of water representing a dilution of $1: 10 ; 5$ c.c. of this mixture was again added to 5 c.c. of distilled water and a dilu tion of 1: 20 obtained; this was again repeated, using 5 c.c. of the mixture last obtained and 5 c.c. of distilled water, and the dilutions were kept up unti a series was obtained representing $1: 10,1: 20,1: 40 ; 1: 80$,
$1: 160,1: 320$, and if necessary $1: 640$ or more. They were then stratified with approximately 1 c.c. of the Wolff phosphotungstic acid reagent, care being taken that the liquids did not mix.

3. This reagent consists of phosphotungstic acid, 0.3 c.c.; conc. Jydrochloric acid, 1.0 c.c.; alcohol, 95 per cent., 20.0 c.c.; distilled viater q. S., 100.0 c.c.
The stomach should be empty. One of us $^{4}$ pointed out elsewhere that even normally a large residue is often found which may in certain pathologic cases assume exaggerated proportions.

In certain experiments (which one of us performed with Drs. Bergeim and Hawk) made in this laboratory, it was remarked that even relatively simple articles of diet such as eggs, combined with cereals and milk, may remain for relatively long periods in the healthy stomach. To commence a test five hours after such a diet will show, even in a certain number of normal individuals, an initial high protein content owing to the dissolved proteins still remaining in the stomach.

Case 23, for instance, demonstrates the results obtained when the test was performed too soon after the individual had ingested a meal of fish and vegetables. In this case the test was purposely performed at that time and the resulting figures show the unmistakable presence of protein residue. If, therefore, high figures are obtained when the stomach should be empty, in the abscnce of other factors which we shall point out, an initial very high point is indicative of protein retention.

Another point is worthy of consideration. The patient is warned to be particularly careful about swallowing oral and bronchial secretions.

An estimation of the protein content of saliva in six $\mathrm{normal}$ individuals showed that it gave a reaction in five cases at $1: 80$ and in one case for some unknown reason at $1: 160$. These figures can be greatly surpassed in the sputum encountered in certain pulmonary cases, and it is an everyday observation that these individuals swallow much of their sputum.

\section{NATURE OF THE PROTEIN REVEALED BY THIS METHOD}

The protein on various occasions found in the gastric juice must come from a. variety of sources. Briefly enumerated it may come from food ingested, saliva or bronchial secretions swallowed, bleeding owing to an ulcerative lesion, regurgitation of intestinal contents, and finally in cases in which there is a gastric lesion, from specific protein products derived from the lesion itself.

That the pure gastric juice derived from normal individuals contains but little protein must be evident from the fact that on various occasions the juice obtained from normal individuals after the administration of a water meal (a test meal consisting of 200 c.c. of water which is aspirated at intervals) never showed a protein content exceeding 1:40. This is significant inasmuch as it indicates that the pure normal juice has a very low protein content, any increase of which must

4. Rehfuss, Martin E., Bergeim, Olaf, and Hawk, Philip B.: The Question of the Residuum Found in the Empty Stomach, THE JourNal. A. M. A., July 4,1914 , p. 11 . 
be of pathologic importance. But the customary Wolff test is performed by administering the ordinary Ewald test-meal and testing at the one-hour interval. Concerning this method we have some data from Wolff, ${ }^{5}$ Einstein, ${ }^{6}$ and Rolph ${ }^{7}$; the results, however, of testing merely one phase of the digestive curve is obviously wrong. It merely registers a single point in the whole mechanism of gastric digestion and gives no information regarding the elaboration of the protein, a point not without importance.

We therefore made studies (1) to determine if possible the actual content of the test meal and its fluctuation during digestion, and (2) the modification of this curve in normal and pathologic cases. These latter are recorded merely in the hope that a possible interpretation may be

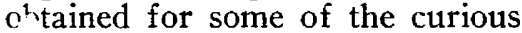
phenomena recorded.

We found that tea alone gave no reaction even at a dilution of $1: 10$, and that a regular Ewald meal consisting of 8 ounces of tea and two pieces of toast gave a reaction at $1: 20$, immediately after thorough maceration. After standing for fifteen hours in the incubator at $38 \mathrm{C} .(100.4 \mathrm{~F}$.) a

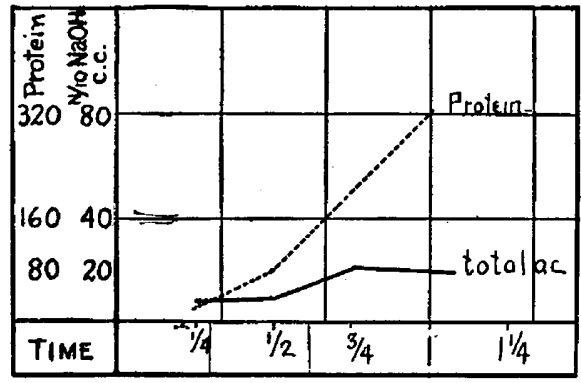

Chart 2.-Protein and total acidity curves in a case of gastric earcinoma.

tcin curve should follow the acid curve and in the absence of acid should remain very low. In other words, depending on the elaboration of acid, there should be a definite elaboration of soluble protein.

\section{DIAGNOSIS OF CANCER FROM BENIGN ACHYLIA BY THIS METHOD}

In an ordinary benign achylia, as seen in Case 5 and in Cases 24, 48 and 49, the albumin curve runs more or less parallel to the acid curve. Even in the subacidities due to benign causes the same rule holds true, and the albumin content remains comparatively low. Smithies, ${ }^{8}$ Wolff ${ }^{5}$ and others found that figures of $1: 100$ were suspicious and above this, point out positive evidence of neoplasm, but the actual evolution of the albumin curve was not worked out. We found that in carcinoma the protein curve diverges from the acid curve, and as digestion progresses this disproportion increases so that there is a marked separation of the curves. This is seen in the cases recorded (Cases 22, 26, 30 and 27 ) in which there was practically an achylia and in which in every instance, subsequent events reaction of $1: 40$ was obtained showing that there was practically no autodigestion. A solution of pepsin without hydrochloric acid gave no protein reaction whatever.

We then performed experiments to study the action of the artificial gastric juice in vitro. The macerated Ewald meal was treated by an artificial gastric juice made by dissolving $0.5 \mathrm{gm}$. of pepsin in 0.2 per cent. hydrochloric acid. The artificial juice failed to give a reaction at $1: 10$. This mixture was then put in the incubator at $38 \mathrm{C}$. and samples were taken at the same time intervals as were employed in taking specimens from the stomach with the fractional tube. The following results were typical of those obtained on three separate occasions:

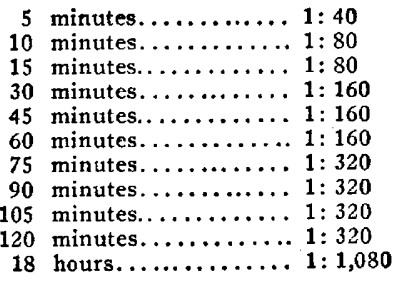

Therefore we see that using this method we obtain a protein in gradually increasing quantities more or less directly proportional to the strength of the acid and the duration of the reaction and directly dependent on the soluble intermediate protein bodies formed through the action of the artificial gastric juice on the proteins of the bread. In each case there was a gradual rise as the continued action of the gastric juice made itself felt on the bread proteins. If, therefore, the gastric mucous membrane is absolutely normal and there are no extrinsic sources of albumin, the pro-

5. Wolff: Berl. klin. Wchnschr., May 29, 1911; March 18, 1912

6. Einstein: Med. Klin., March $24,1912$.
7. Rolph: Med. Rec., May 10, 1913, p. 848. and roentgenoscopy confirmed the diagnosis that the protein curve was entirely disproportionate to the acid curve. Secondly, that this increase in albumin concentration occurred as digestion proceeded suggested that there was an elaboration of protein on the part of the neoplasm per se. In other cases in which the neoplasm was accompanied by a demonstrable acidity, the protein was generally high with a tendency to become higher as digestion proceeded. Protein retention will result in high initial figures, but if the precaution is taken to empty the stomach before testing, this factor is disposed of. In the question of the differential diagnosis between cancer and benign achylia we wish to report two cases of considerable interest, which show how important it is to interpret the findings correctly.

CASE 12.-A man, aged 44 suffering from gastric disturbances for many years, showed in the last few months before admission rather marked anorexia, profound anemia, a tendency to daily vomiting of yellowish material and mucus. He has lost distinctly in weight, and his anemia left him very weak, with paresthesias of the hands and feet. With the exception of intervals of mucus colitis, he has had rather marked constipation. The expectoration, which is pronounced, is free from tubercle bacilli, but contains many staphylococci, Micrococcus catarrhalis and a streptococcus. Physical examination as well as roentgenography fail to reveal anything in the lungs except a little thickening of the hilus of the right lung. From the character of the sputum, its persistence and the physical findings, we still thought (with Dr. Beardsley and Dr. Tracey of Beverley, to whom we were indebted for the case) that there must be bronchiectasis. Examination of the blood showed profound

8. Smithies: Am. Jour. Med. Sa, May, 1914, p. 713. 


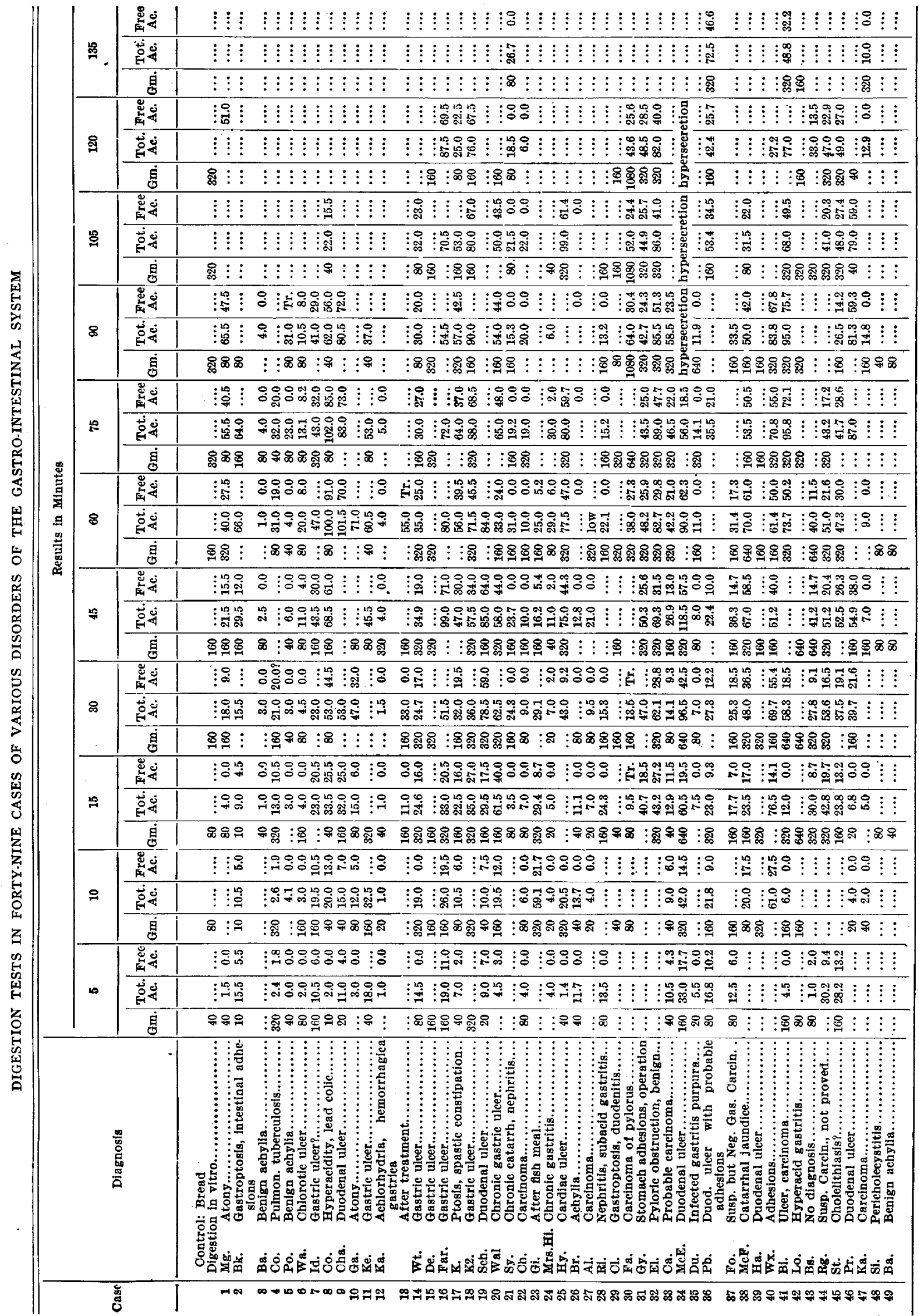


anemia, but none of the true findings of pernicious anemia. An analysis of the gastric juice showed a total achylia persisting throughout the entire digestive cycle and absolutely no ferment response, occult blood positive, many smaller micro-organisms, but no lactic acid and no Oppler-Boas bacilli. The protein result is shown in No. 12 in the table. The sudden jump was synchronous with the appearance of bleeding and therefore was not due to the elaboration of the protein through a specific neoplasm, but to the protein in the blood exuded. Two things decided us against the diagnosis of neoplasm-first, the long duration of the case; secondly, the absence of any Roentgen-ray or palpatory evidence in the face of such a pronounced gastric finding. The case, which will be reported, is of the greatest interest. Suffice it to say, that by means of intragastric, dietetic and certain internal glandular medication, there was steady improvement with a disappearance of the gastric symptoms. A vaccine was made for the expectoration, and at this writing, after the fifth administration of the vaccine, the expectoration has disappeared, and the patient is 3 pounds over his best weight.

This case demonstrates forcibly the danger of taking the protein content at a given point and deciding for or against carcinoma of the stomach. Case 35 is similar, a direct infection of the stomach in a subject with purpura hemorrhagica, in which the presence of blood has caused a marked increase in the protein content. But in carcinoma, the protein continues to increase until the end of digestion. Case 22 is one in which there was a large inoperable carcinoma in the wall of the stomach. Case 27 is a similar case showing a gradual, steady and exaggerated rise in protein content. Case 30 shows to what extent this might go in carcinoma of the pylorus. In this case the retention liquid in the empty stomach was filled with a purée of leukocytes amounting to diluted pus; this was undoubtedly due to the ulceration of the neoplasm.

\section{DIAGNOSTIC VALUE OF THE PROTEIN CONTENT IN} ULCERATIVE CONDITIONS OF THE STOMACH

We made a series of protein determinations on cases of clinically diagnosable ulcer, all of which had been submitted to careful physical examination and in most cases, roentgenoscopy. We found an altogether unusual protein curve in these cases, namely, a rapid rise in the protein concentration out of all proportion to the protein elaborated by the action of any juice in this period of time, arguing, therefore, for an extrinsic cause for the protein. This is to be seen in practically all cases on this list in which the diagnosis of ulcer was made, and the type of the protein curve in contrast to the acid curve is striking. Case 6 shows the very early initial rise. This was an undoubted case of gastric ulcer, showing the characteristic tender point, pain, and occult blood in both gastric contents and feces, and marked secondary anemia. The presence of the occult blood may be the cause for the phenomenon. In Case 8, which several clinicians thought one of gastric ulcer, the absence of characteristic symptoms, the absence of the minutest traces of bleeding, and the character of the curve enabled us to rule out ulcer. This was justified by the subsequent course of the case.
Case 11, although the data are not complete, is in all probability one of ulcer, there being occult blood at times in the movements on a meat-free diet. Case 14 shows the recurrent pain, characteristic tender point, slight tendency to rigidity of the upper right rectus, persistent history of ulcer, and alimentary hypersecretion. Case 15 shows the persistently high protein content from a case of bleeding ulcer. The history is of long duration, characteristic pain, hematemesis, tender point, occult blood in the gastric contents and stools, and the early symptoms of gastric retention. Hypersecretion followed the meal and was pronounced. This liquid, which was found free from food residues, showed a protein content of $1: 160$, entirely different from the normal gastric juice. This was salted out with ammonium stlphate and filtered, and the albumin content dropped to $1: 40$, suggesting very strongly serum albumin probably derived from blood, as. the cause of the high content. Case 16 was a typical case of ulcer with two severe attacks of hematemesis very large in amount, characteristic pain, both alimentary and continued hypersecretion, a tender point, and profound anemia from hemorrhage. Case 18 is one of pyloric ulcer with obstruction and retention, showing the very high point from the very first sample, undoubtedly due to protein retention. Cases 9 and 19 were cases of duodenal ulcer. Case 20 alone was puzzling, characteristic ulcer symptoms alternating with periods of relief. The case was most likely ulcer, high acid figures and hypersecretion playing an important part, although the evidence here renders the diagnosis uncertain. These cases are merely presented as showing a striking protein curve in cases of ulcer. Whether this is a constant finding, we are not prepared to state. We merely wish to submit these results for further investigation, believing that the high protein curve which does not occur in ordinary cases suggests the possibility of ulcer. As to the cause of this rise, whether it is due to bleeding, or hypothetically to exudation of protein products from the ulcer region, or to the action of the corrosive juice on the ulcer, we cannot say. Some further results seem to show that while the high protein content remains sustained in gastric ulcer, in duodenal ulcer there is an initial rise which apparently falls off more rapidly, possibly owing to the hypermotility encountered in a certain proportion of the cases of the latter disease. As yet we have insufficient data to make a definite statement, but in those cases in which the diagnosis was made the rise of the curve to its high point was slower.

\section{METHOD OF INTERPRETATION OF THE RESULTS OF THE REACTION}

In interpreting any protein curve, the question arises as to the quantity and source of the protein. We have pointed out that, normally, in the absence of any extrinsic factor, the quantity of dissolved protein runs more or less parallel to the duration of time in the stomach and the acidity. Any marked deviation nitust 
therefore be accounted for. On the basis of this finding rests the value of the test for diagnostic purposes. If the protein curve simply follows the acid curve, it merely represents the action of the gastric juice on the bread ingested, and may be taken as an index of gastric function. This is already given in the acid curve. But abnormal or pathologic possibilities exaggerate the curve out of all proportion to the acid curve, either in the very beginning, during, or at the termination of the curve. Therefore, if bread alone can only give us a definite amount of protein within a definite time, an exaggerated quantity or the presence of high figures must mean that the protein comes from other sources than bread. In the present work, the separation and recognition of the different forms of protein has not seemed practical for routine work. The method of interpretation has been based on a comparison of the acid and protein curves. It is therefore perfectly evident that a certain protein concentration in a given time can be produced from a standard meal. What are the extrinsic sources of protein? These may be (1) blood; (2) the presence of pus either intragastric in origin or swallowed; $(3)$ the end-products of protein digestion still in the stomach through atony or obstruction; therefore stasis, lack of motor tone or actual obstruction may unduly increase the protein concentration, all of which are removed by emptying the stomach before administering the meal; (4) a possible exudation of lymph or serum from ulcer; (5) the exudation from a malignant lesion. These factors should be borne in mind in interpreting the findings. In Cases 2, 8, 1, 10, 13, the findings indicated the absence of ulceration, and the subsequent course of the

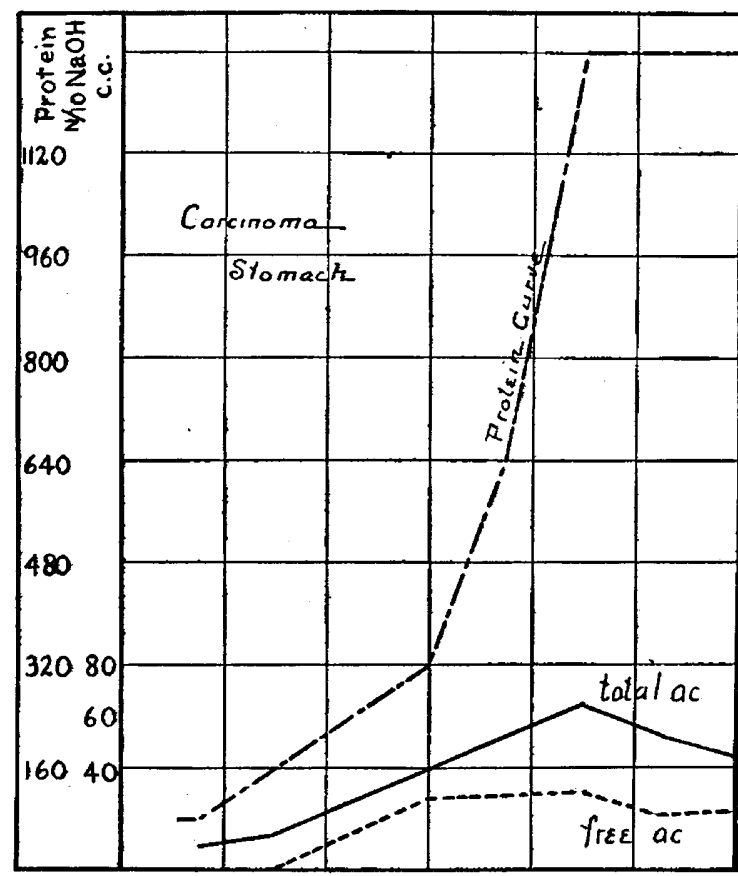

Chart 5.-Protein, total acidity and free acid curves in case of gastric carcinoma. food residues, or the swallowing of protein material such as certain forms of sputum.

3 . Bread and tea alone, following the composition of the Ewald meal, will show in the absence of any pathologic factor a definite amount of protein corresponding to the curve or the digestive power of the juice secreted. A mixture or maceration of bread in tea will show a protein content on 1:20-1:40; if the mixture is acted on by an artificial gastric juice in vitro the protein content of the juice rises steadily within the next two hours and may reach 1:320 in seventyfive minutes. - In other words, there is a transformation and liberation of soluble protein which may bè demonstrated by the Wolff technic.

4. The pathologic significance arises when a curve shows any marked deviation from this recognized standard, that is to say, when there is an undue concentration of protein out of all proportion to that normally found at that particular phase in digestion. If therefore a marked increase in protein does not conform in a general way to the acid curve it can be definitely stated that the protein is coming from other sources than the proteins of the bread.

5 . An analysis of the protein would seem to demonstrate that normally it is of the nature of a proteose, but in inflammatory or ulcerative conditions it is probably serum protein removed to a large extent by saturation with ammonium sulphate.

6. We could call attention to the interesting curves found in ulcer, many of which showed traces of blood, several of which can be explained on the basis of protein retention, some of which must be explained on the basis of hypothetic exudation.

7. In the differentiation of achylia and carcinoma, cases demonstrated the correctness of our diagnosis. In Case 14 the curve indicated strongly the presence of ulcer which would cover all the symptoms of the case. In Case 24, the diagnosis of neoplasm was definitely eliminated on that finding and subsequent events justified the diagnosis. In Case 27, it is evident that an achylia could not produce such a curve unless an added factor such as the ulceration of a neoplasm came into play. In conclusion, we may state that while we do not claim any pathognomonic value for the test, we de believe that it will add another link to the chain of evidence which may decide for or against a given diagnosis.

\section{CONCLUSIONS}

1. The gastric juice in health shows definitely a protein content of very low degree.

2 . This content is increased in disease by the addition of an exudation of protein material from inflammatory, ulcerous, or carcinomatous mucous membranes, or by the addition of partially digested and retained proportion as the case approached a true achylia and the added factors (extrinsic) such as swallowed pus, bleeding and protein residues could be ruled out. We likewise pointed out that the one-hour point was insufficient for examination and that the characteristic for carcinoma in these cases is a divergence of the protein curve out of all proportion to the acid curve. Infected catarrh, hemorrhagic erosions, achlorhydria, hemorrhagica gastrica, may give high findings, but they do not have the tendency to give a steadily increasing protein content.

8. We believe that a study of the protein curve may yield information of the greatest value, provided that all the precautions have been observed.

Spring Tonics.-The idea that a "tonic" is needed in the springtime is a mere tradition. Most of such tonics sold to the public are strongly alcoholic, and may prove distinctly harmful.-Health Letter, Life Extension Institute. we pointed out that the test was of value in direct 